\title{
Thromboprophylaxis for Hospitalized Medical Patients: A Multicenter Qualitative Study
}

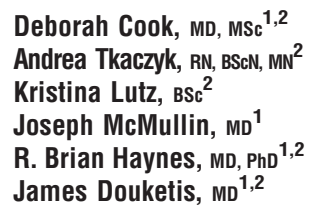

\author{
${ }^{1}$ Department of Medicine, McMaster University, Hamilton, Ontario, Canada. \\ ${ }^{2}$ Department of Clinical Epidemiology \& Biostatistics, McMaster University, Hamilton, Ontario, Canada.
}

This study was funded by a Team Grant from the Canadian Institutes for Health Research led by Dr. Jeff Weitz. Dr. Cook is a Canada Research Chair of the Canadian Institutes for Health Research. Dr. McMullin holds a Partnership Award with St. Joseph's Healthcare and the Canadian Institutes for Health Research.

Disclosure: Nothing to report.

BACKGROUND: Observational studies have documented that medical patients infrequently receive venous thromboembolism (VTE) prevention.

OBJECTIVE: To understand the barriers to, and facilitators of, optimal thromboprophylaxis.

PATIENTS: Hospitalized medical patients.

DESIGN: We conducted in-depth interviews with 15 nurses, 6 pharmacists, 12 physicians with both clinical and managerial experience, and 3 hospital administrators.

SETTING: One university-affiliated and 2 community hospitals.

INTERVENTION: Interviews were audiotaped and transcribed verbatim. Transcripts were reviewed and interpreted independently in duplicate.

MEASUREMENT: Analysis was conducted using grounded theory.

RESULTS: Physicians and pharmacists affirmed that evidence supporting heparin is strong and understood. Clinicians, particularly nurses, reported that mobilization was important, but were uncertain about how much mobilization was enough. Participants believed that depending on individual physicians for VTE prevention is insufficient. The central finding was that multidisciplinary care was also perceived as a barrier to effective VTE prevention because it can lead to unclear accountability by role confusion. Participants believed that a comprehensive, systems approach was necessary. Suggestions included screening and risk-stratifying all patients, preprinted orders at hospital admission that are regularly reevaluated, and audit and feedback programs. Patient or family-mediated reminders, and administrative interventions, such as hiring more physiotherapists and profiling thromboprophylaxis in hospital accreditation, were also endorsed.

CONCLUSIONS: Universal consideration of thromboprophylaxis finds common ground in multidisciplinary care. However, results of this qualitative study challenge the conviction that either individual physician efforts or multidisciplinary care are sufficient for optimal prevention. To ensure exemplary medical thromboprophylaxis, clinicians regarded coordinated, systemwide processes, aimed at patients, providers, and administrators as essential. Journal of Hospital Medicine 2009;4:269-275. @ 2009 Society of Hospital Medicine.

KEYWORDS: heparin, medical patients, prevention, prophylaxis, thrombosis.

Venous thromboembolism (VTE) is a common complication of serious illness, conferring increased morbidity and mortality in hospitalized medical patients. Thromboprophylaxis has been rated the number 1 patient safety intervention for hospitalized patients by the Agency for Healthcare Research and Quality, supported by evidence of effectiveness in multiple methodologically rigorous randomized trials. ${ }^{1}$ Unfortunately, many studies have shown that suitable patients do not receive thromboprophylaxis when they should. For example, in a large teaching hospital, 44 of 245 VTE events were considered to be potentially preventable, occurring because of omitted prophylaxis, inadequate duration of prophylaxis, or incorrect type of prophylaxis. ${ }^{2}$

Medical patients appear to be at particularly high risk of not receiving thromboprophylaxis. One retrospective study of 446 medical patients in 2 hospitals revealed that only $33 \%$ had appropriate prophylaxis. ${ }^{3}$ In a large retrospective study of 29 Canadian hospitals, among 1,894 patients for whom thromboprophylaxis was considered necessary, only 23\% received it. $^{4}$ These findings are comparable to other practice audits $^{5-12}$ and a large international cross-sectional study $^{13}$ showing that appropriate thromboprophylaxis is 
administered to only 1 of every 3 hospitalized medical patients eligible for prophylaxis. Furthermore, of patients diagnosed with VTE in a large international registry, only $33 \%$ had received heparin thromboprophylaxis prior to their event. ${ }^{14}$

The objective of this study was to understand the barriers to, and facilitators of, optimal thromboprophylaxis in hospitalized medical patients using grounded theory methods. To address our poor understanding of low rates of thromboprophylaxis on hospital medical wards, we used qualitative research, which focuses on social and interpreted, rather than natural and objectified, phenomena. Qualitative research also aims to discover, describe, and understand, rather than to test and evaluate. ${ }^{15}$ Our research question was, "What do healthcare clinicians, managers and hospital administrators perceive inhibits the implementation of thromboprophylaxis for medical patients, and what do they perceive would help to optimize thromboprophylaxis?"

\section{Methods}

We conducted the Qualitative Thromboprophylaxis Enquiry of Compliance (QUALITEC) survey at 3 institutions from June 2006 to September 2007. One was a university-affiliated hospital (St. Joseph's Healthcare, Hamilton, Ontario, Canada); the other 2 were community hospitals (Joseph Brant Hospital, Burlington, Ontario, and Credit Valley Hospital, Mississauga, Ontario, Canada).

Participants included: (1) bedside nurses, nurse clinicians, and nurse educators; (2) pharmacists; (3) attending physicians; (4) nurse, pharmacy, and physician managers; (5) hospital administrators; (6)medical residents; and (7) members of the hospitals' Quality Improvement Team. Participants in groups 1 to 3 were persons who had cared for medical patients in the index hospital for at least 2 years. Participants in groups 4 and 5 were persons who had worked in the index hospital for at least 2 years. The requisite minimal duration of work on the medical ward for residents was 1 month. There were no exclusion criteria for participants based on sex, religion, ethnicity, or culture. Thus, we enrolled participants based on their a priori eligibility criteria ("criterion sampling") and their ability to allow us to achieve our objectives ("purposive sampling").

The Research Coordinator created a list of potential interview candidates by examining the organizational structure of each hospital to identify the names of clinicians, managers, and administrators who had responsibility for medical patients. This person identified the names of potential medical ward nurses through discussion with the nurse manager of each medical unit and identified the names of potential attending physicians, residents, and pharmacists through discussion with the physician director or designate of the medical unit ("snowball sampling").

After piloting testing with 2 trial participants, the Research Coordinator conducted in-depth, open-ended, 1- on-1 interviews using a flexible interview guide. This approach allows respondents to use their own words to express ideas, and affords opportunities to probe for more information through dialog. All potential participants were provided with a brief study summary. Each interview took approximately 30 to 60 minutes. All interviews were audiotaped and transcribed verbatim. The Research Coordinator wrote field notes on the main concepts featured in the interview.

To characterize the participants, we recorded their age, sex, position, number of years in their current position, number of years in their profession, and number of years working in their hospital. For clinicians, we recorded the proportion of their professional time spent in clinical practice, the proportion of their clinical time spent caring for hospitalized medical patients (on the medical wards, doing consultations, and in the emergency department), and whether they had a formal role in a previous quality improvement project.

We transcribed each interview upon completion. As is typical for qualitative research, we began data analysis during the data collection phase. Transcripts were analyzed using the coding methods described by Strauss and Corbin. ${ }^{16}$ The main content analysis of QUALITEC consisted of line-by-line open coding. All transcripts were coded by 2 investigators independently who created categories and themes. The first coding process of grounded theory analysis was reduction of data to identify categories (experiences and actions that were similar or related) and to define their dimensions. We iterated between data collection and analysis, revising the interview guide to refine questions and focus on unique concepts. The qualitative data management software $\mathrm{NVivo}^{\mathrm{TM}}$ helped with linking codes and categories to ultimately organize ideas into a main theme and several key concepts; it also helped with retrieval of specific quotes. A third investigator reviewed the transcripts independently to identify categories. Analysis continued while the study progressed until saturation of information occurred and no new categories emerged. We invited 3 participants to review an early draft report ("member checking”).

\section{Research Ethics}

This study was approved by the Research Ethics Board at each participating center. Written informed consent was obtained from each participant. Participation was voluntary and confidential; participants had the opportunity to withdraw from the study without any consequences. Data were deidentified upon transcription and remained anonymized, while kept in secure password protected computerized files. Participants received a $\$ 25$ gift certificate as a token of appreciation. We conducted this study according to the Guiding Ethical Principles section of the Tri-council Policy Statement of Ethical Conduct for Research Involving Humans. ${ }^{17}$ 


\section{TABLE 1. Characteristics of Study Participants}

\begin{tabular}{lc} 
Characteristic & \multicolumn{1}{c}{ Value } \\
\hline Female, $n$ (\%) & $27(75.0)$ \\
Age, mean (SD) & $39.6(15.6)$ \\
Hospital, $n$ (\%) & \\
St. Joseph's Healthcare, Hamilton & $16(44.4)$ \\
Joseph Brant, Burlington & $9(25.0)$ \\
Credit Valley, Oakville & $11(30.6)$ \\
Years in hospital, mean (SD) & $10.1(7.4)$ \\
Position, $n$ (\%) & \\
Bedside nurse & $8(22.2)$ \\
Charge nurse & $1(2.8)$ \\
Nurse clinician & $2(5.6)$ \\
Nurse educator & $2(5.6)$ \\
Nurse manager & $2(5.6)$ \\
Pharmacist & $3(8.3)$ \\
Pharmacist manager & $3(8.3)$ \\
Resident physician & $4(11.1)$ \\
Attending physician & $6(16.6)$ \\
Physician manager & $2(5.6)$ \\
Quality improvement team member & $2(5.6)$ \\
Hospital administrator & $1(2.8)$ \\
Years in current position, mean (SD) & $7.3(6.4)$ \\
Participated in quality improvement projects, $n$ (\%) & $20(55.6)$ \\
Clinical focus (for clinicians only), mean (SD) & \\
Time spent in clinical practice (\%) & $93.6(13.4)$ \\
Time spent caring for medical patients (\%) & $79.2(25.1)$ \\
\hline
\end{tabular}

NOTE: Data for $\mathrm{n}=36$ study participants.

Abbreviation: SD, standard deviation.

\section{Results}

Of 39 persons we approached to participate, 36 out of 39 (92.3\%) agreed. Two nurse managers at 1 community hospital declined to participate. One resident was missed due to clinical responsibilities. In Table 1, we present the characteristics of the 36 participants. When asked about the level of concern about thromboprophylaxis on the medical wards, participants stated that clinicians were generally insufficiently concerned $(24 / 36,68.6 \%)$ or appropriately concerned $(11 / 36,31.4 \%)$.

\section{Barriers}

Relying on individual physicians for VTE prevention was regarded as ineffective, since medical patients often do not receive prophylaxis when they should.

It's sort of Russian roulette as to whether or not we grab a patient and it actually gets done for them. [bedside nurse]

There's a lot of mavericks out there. They do their own thing, and it has never been a problem. They're not held accountable. [quality improvement team leader]

Several different clinician groups were regarded as being involved in medical thromboprophylaxis.

Physiotherapy, occupational therapy, doctors, hopefully our clinical care educators, charge nurses .... I think pretty much every-

body's involved .... I f feel that's part of my role as care giver. [bedside nurse]

Many people in the healthcare team are involved in [deep vein thrombosis] DVT prevention ... at the nurse level, physiotherapy, occupational therapy (in terms of mobility of the patient) ... the physician, specialty services like thrombo service, hematology department. [nurse manager]

Since many clinicians implicitly or explicitly have a role in thromboprophylaxis, some participants viewed it as everyone's responsibility, or that it should be everyone's responsibility, to ensure optimal VTE prevention.

I think the responsibility should be everyone's responsibility-all across the health care professions. [ward pharmacist]

Make it so that you have a few stopgaps so that a few people are looking for the risk. . . and make the process different so that you have a couple of stops along the way that it'll get brought up. .. . I would create it so that it's not just one group in charge of the DVT prophylaxis decision and assessment. It has to be across the board. [nurse educator]

However, while multidisciplinary care was considered ideal to achieve optimal patient outcomes, it was also paradoxically perceived as insufficient to ensure effective thromboprophylaxis. Multidisciplinary care can lead to confusion about roles on a team, and unclear accountability, thereby becoming a potential barrier to effective prevention. Some participants thought that just one person should be ultimately responsible for thromboprophylaxis.

I think it would be crucial to identify one person responsible rather than indirectly a number of people who would be encouraged . . . . It is probably better if you make one of those targets dependable. [physician]

[If] you assign it to one person versus having the accountability spread among [all] those people - then nobody takes accountability. [quality improvement team member]

Many participants reported that mobilization was important, though difficult to achieve. They also expressed uncertainty about whether, and how much, mobilization is enough.

I think the best way to prevent DVT would be to fully focus on early mobilization, regular physiotherapy, and how a patient returns to regular activities to get them up and around. [resident]

Level of mobility is a subjective matter. . . When it comes to the question of "Well, how mobile is mobile enough?", that's not standardized to my knowledge and is very subjective as far as when to stop it. Is getting up in a chair and wiggling your toes good enough?. . . Or are they running up and down the hall and you have to chase them? Really, what level of mobility is considered the standard for discontinuing DVT prophylaxis? That's the question that comes up repeatedly so I think that's a very large barrier. [pharmacy manager]

Several logistic barriers associated with antiembolic stockings and pneumatic compression devices were cited, 
including problems with fit, inconvenience, noncompliance, and cost.

I find stockings aren't always measured or worn appropriately. It's difficult; I think every nurse measures them differently. If they're too tight around the thighs they just roll them down. If anything, they really constrict any type of circulation rather than promote it. Moon boots . . . are all right, but a little cumbersome, more expensive and ... are more geared to specific patients. [bedside nurse]

The patients want them off at certain times of the day. Theoretically, you only take them off to bathe them but some of them are really bothered by them. [nurse clinician]

Another key barrier was that clinicians are more focused on treating the immediate health care problem precipitating hospital admission than on preventing future complications.

It's something that we tend to forget because patients come in with giant medical problems a, b, and c, and then your DVT prophylaxis tends to fall by the wayside, as you're trying to deal with their major medical issues. [resident]

Prevention issues are a little bit different than treatment issues . . . . We see ourselves as 'interventionists' more than "preventionists". . .. It's the medical things we tend to deal with immediately and that's often the focus . . . why the patient is in hospital, rightly or wrongly. Quality ... doesn't just include intervention, it includes prevention. [nurse manager]

\section{Potential Solutions}

To address this problem of inattention to preventive strategies, participants indicated that local data on the burden of illness and current utilization of thromboprophylaxis would be helpful. Some participants described institutionspecific information and thromboprophylaxis targets that motivate clinicians.

Give feedback to the team and physicians about what the incidence of DVT is. I don't think everybody knows. I don't even know in our hospital what it is. So give feedback to the team about what the incidence of DVT is, how many people are on DVT prophylaxis .... and how many people die from pulmonary embolism. If you have those numbers in front of you, then you would have something to aim for. [physician manager]

You do run charts so that you are auditing continuously and reporting the results back to the staff. And we have weekly meetings with staff members. That has been effective. And we have tip of the week via email. [quality improvement team member]

Most participants recommended redoubling efforts towards anticoagulant thromboprophylaxis-a coordinated, systemwide approach across the continuum of care.

It's got to be a trigger for every patient that comes in. Does this patient need DVT prophylaxis? Is this patient a candidate for it? [physician manager]

I think if it's not tackled at the beginning it gets lost in the shuffle. I think if it's something we put into place just like we do when we're getting a history and on anything else. If we start with it [heparin] from day one we'll continue it through right to the end. [bedside nurse]
Participants suggested a variety of methods to enhance thromboprophylaxis. These included a universal, structured, dynamic risk stratification tool, and standardized order forms at the time of hospital admission, which would be reevaluated regularly, and require an enabling educational program.

Every medical patient 18 or older will be given a risk score. Risk stratifying all of our patients with just a simple little tool and considering treatment for those that are of high risk is what we need to do. Reinforcing the education and teaching required for the patients that are at low risk, because we don't need to put everyone on heparin. But anyone could potentially get a clot. [nurse educator]

It could be a standardized order sheet for every admitted patient and maybe that could help. And that would be like the first page in every chart in the whole hospital, and they can always cross it off and say I don't want to use this. [ward pharmacist]

The hospital intranet was sanctioned as a useful repository for physician orders and other tools. Computerized health records and computer decision supports were strongly endorsed.

It should be in a computerized system . . . if it goes into a manual system, paperwork tends to get lost. I think a computerized system would be ideal. [pharmacist]

I think that having a computer health record-that we would love, for many reasons-once that happened, it would be a way to ensure it, because there would be prompts . . . . You would get a prompt saying, Why are you not using it? . . . . An electronic health record and as a back up, having a pharmacist. [physician]

Leveraging patient or family-mediated interventions to provide reminders was also suggested, given the familiarity of the public with thrombosis.

Leave every patient a small pamphlet saying "Are you getting DVT prevention? Are you getting injections?" Empower people as well. [physician]

I think the absolute biggest driver from our perspective in admin is always public awareness. The demand for standard service increases the most when the public is aware of it. . . They ask. Patients are becoming more educated, they use the Internet, they search those things out themselves and they are knowledgeable. [hospital administrator]

Sufficient human resources to ensure mobilization and profiling thromboprophylaxis during accreditation were regarded as administrative initiatives that could help. Capitalizing on social forces in healthcare such as patient safety could also galvanize efforts to prevent VTE.

We need physio and [occupational therapy] OT. We need more rehab. We need resources. That's what we're lacking. The only physio and OT that comes to our floor is pending discharge. So it's definitely resource-related. [bedside nurse]

From an administrative perspective, the whole concept of preventing complications reduces risk, improves patient safety, reduces length of stay-all those warm fuzzy things that are attached to providing the best possible care for the patient at the right time. [nurse manager] 


\section{Discussion}

In this qualitative study, participants affirmed that depending on individual physicians for VTE prevention is insufficient. Distinct from most therapeutic interventions which are understood as the responsibility of physicians, preventive interventions such as thromboprophylaxis may be more readily embraced as the charge of members of a multidisciplinary team. While every clinician group felt compelled to help with VTE prevention, reliance on multidisciplinary care was also perceived as a barrier to effective VTE prevention because it can generate confusion about roles. Participants recommended a comprehensive, systems-approach, including screening and risk-stratifying all patients, preprinted orders at hospital admission that are regularly reevaluated, and audit and feedback programs. Also endorsed were patient or family-mediated reminders, and administrative interventions such as hiring more physiotherapists for mobilization and profiling thromboprophylaxis for hospital accreditation.

Approximately $70 \%$ of participants judged that clinicians were insufficiently concerned about thromboprophylaxis. In contrast to many commonly cited reasons for underutilization of evidence-based interventions such as lack of awareness of (or resistance to) new information, and lack of selfefficacy of clinicians (wondering whether the benefits observed in the research setting will be realized in the practice setting), ${ }^{18}$ participants in our study did not raise these as barriers to thromboprophylaxis. As physician and pharmacist participants indicated, because the evidentiary basis for heparin thromboprophylaxis is strong and understood (in contrast to that for mechanical prophylaxis), lack of knowledge was not considered a barrier.

Our findings are consistent with a previous qualitative study in which clinicians and managers were interviewed to learn about factors that increase beta-blocker use following myocardial infarction; these investigators found that administrative support, use of data, and quality improvement initiatives were key. ${ }^{19}$ Detailed implementation directives to clarify clinical responsibilities for many different stakeholders were also suggested in a qualitative study on the optimal use of noninvasive ventilation..$^{20}$ Extending these results, participants in our study suggested several interventions aimed at different levels of the healthcare system (eg, patient, provider, and administrator) that are either enabling or reinforcing. While just 1 implementation strategy may result in improved thromboprophylaxis, ${ }^{21}$ efficient application of multiply-redundant strategies delivered by a multidisciplinary team may be more powerful. Such an approach has driven high level performance for the American Heart Association's "Get With The Guidelines" Program, resulting in lower community and hospital cardiovascular and cerebrovascular mortality in Massachusetts. ${ }^{22}$

Although our aim was to obtain multidisciplinary input, we did not focus on physicians who primarily prescribe thromboprophylaxis, thereby potentially underrepresenting their views relative to their role. We did not interview all clinicians who can influence VTE risk (eg, physiotherapists) or prescribe thromboprophylaxis (eg, family physicians). During this study, patient safety emerged as a major hospital initiative, ${ }^{23}$ which could modify participants' views on the importance of VTE prevention. Although VTE prevention may be enhanced by understanding barriers to, and solutions for, optimal thromboprophylaxis from both clinicians' and managers' perspectives, the suggestions we elicited on methods to improve VTE prevention represent participants' opinions, rather than evidence, about effective strategies. Our findings are not generalizable to settings with nurse practitioners who are dedicated to VTE prevention, nor to settings with computer decision support systems that already incorporate thromboprophylaxis.

Strengths of this study include interviewing key stakeholders representing several clinician groups and managers, to obtain perspectives on both individual and systemwide influences on thromboprophylaxis in medical patients. By eliciting the views and experiences of participants in both the community and university settings, we captured multicenter perspectives on preventive health. We used triangulation of data sources (researcher and participant) and invited participants to review an early draft of the report (member checking) ${ }^{24}$ This study highlights the merits of qualitative research which can provide insights into familiar patterns and problems, and contribute to knowledge for interdisciplinary audiences. These qualitative research results help to explain quantitative research results documenting very low rates of medical thromboprophylaxis, ${ }^{2-14}$ raising hypotheses to test in future studies testing systemwide interventions that may improve patient safety. One prominent publication reported the impact of electronic reminders, ${ }^{25}$ while a recent systematic review of methods to increase thromboprophylaxis outlines many potentially effective approaches. ${ }^{21}$

In summary, the findings of this qualitative study challenge the notion that either individual physician efforts or multidisciplinary care are enough to lead to optimal VTE prevention. Participants believed that while well-functioning teams at the bedside hold great promise to deliver superior care, they may also lead to unclear role definition. Since physicians commit many errors of omission regarding thromboprophylaxis, these results raise the possibility of delegated medical acts outside the scope of conventional practice for nurses and pharmacists. Leveraging the skills and knowledge of multidisciplinary teams for medical thromboprophylaxis requires not only clear accountabilities, but also excellent interprofessional communication, and institution-wide approaches to change prescribing behavior, potentially involving patients and administrators as well as clinicians.

\section{Appendix: Qualitec Interview Guide}

\section{Opening Statement}

Thank you for your participation in this research study we are conducting with colleagues at St. Joseph's Hospital and 
McMaster University. Through this interview, I hope to learn how you think about DVT prevention in medical patients. When you answer the interview questions, please keep in mind that we are interested in your own thoughts and approaches.

First, I have a consent form I'd like you to read to make sure you understand the reason for this study, and to see if you have any questions about becoming involved. Please take a few minutes to read it over.

[Participant reads consent, questions about the study may or may not ensue, participant signs to indicate informed consent, the interview proceeds.]

\section{Structured Interview Begins \\ Demographics}

Before we begin the interview, I would like you to take a few minutes to complete this form, which consists of a few demographic questions.

[Participant completes demographic form].

Thank you. Now I'll move on to some questions about DVT prevention.

[The interviewer informs the participant that she is putting the tape recorder on].

\section{Core Study Interview Guide}

1. Can you tell me a bit about your own approach to DVT prevention in medical patients? For what types of patients are you concerned about DVT?

2. What do you find are the best ways to prevent DVT? Why?

3. Who else is involved in trying to prevent DVT? In general, how are DVTs are prevented at this hospital?

4. What are some of the barriers to preventing DVTs? (e.g., risks of prevention, disbelief that prevention really works, DVT prevention is someone else's responsibilitywhose?)

5. Are there other ways to ensure that DVTs are prevented in medical patients? If so, how would they work?

6. Can you tell me how different clinicians (such as physicians, nurses, pharmacists, and residents) could do a better job at preventing DVT? How could medical wards and departments help? How could hospital administration help?

7. Do you remember a patient who had a problem with DVT prevention? If so, can you tell me about this problem and how it might have been avoided?

8. If you could create a perfect system of DVT prevention what would it be?

9. Do you think people are overconcerned, appropriately concerned, or underconcerned about DVT prevention?

10. Is there anything else you would like to tell me?

\section{Final Debriefing Question}

How has this interview experience been for you? Thank you very much for sharing your views. We really appreciate it.

\section{Acknowledgements}

The authors thank the participants of this study for sharing their experiences and views with them. The authors are grateful for the help of Laurel Raftery, Michelle Murray, Monica Owen, and Shelley Anderson for transcriptions. The authors appreciate the organizational assistance of Nancy Lloyd and the biostatistical support of Diane Heels-Ansdell for analyzing respondent characteristics. The authors are grateful to Nancy Lloyd, Alice Huh, Barbara Young, and Gray Ellrodt for feedback on earlier versions of this report. Deborah Cook had full access to all of the data in the study and takes responsibility for the integrity of the data and the accuracy of the data analysis.

\section{Address for correspondence and reprint requests:}

D.J. Cook, Departments of Medicine \& Clinical Epidemiology, McMaster University Medical Centre, 1200 Main St West, Hamilton, Ontario, Canada; Telephone: 905 525-9140 X23162; Fax: 905524 3841; E-mail: debcook@mcmaster.ca Received 23 July 2008; revision received 21 October 2008; accepted 12 November 2008.

\section{References}

1. Agency for Health Care Policy Research. Prevention of venous thromboembolism after injury. Summary, evidence report/technology assessment. Available at: http://www. ahrq.gov/clinic/epcsums/vtsumm.htm. Accessed April 2008.

2. Arnold DM, Kahn S, Shrier I. Missed opportunities for prevention of venous thromboembolism: an evaluation of the use of thromboprophylaxis guidelines. Chest. 2001;120:1964-1971.

3. Rahim SA, Panju A, Pai M, Ginsberg J. Venous thromboembolism prophylaxis in medical inpatients: a retrospective chart review. Thromb Res. 2003;111:215-219.

4. Kahn SR, Panju A, Geerts W, et al., for the CURVE Study Investigators. Multicenter evaluation of the use of venous thromboembolism prophylaxis in acutely ill medical patients in Canada. Thromb Res. 2007;119: 145-155.

5. Proctor MC, Greenfield LJ. Thromboprophylaxis in an academic medical center. Cardiovasc Surg. 2001;9:426-430.

6. Ahmad HA, Geissler A, MacLellan DG. Deep venous thrombosis prophylaxis: are guidelines being followed? ANZ J Surg. 2002;72:331-334.

7. Aujesky D, Guignard E, Pannatier A, Cornuz J. Pharmacological thromboembolic prophylaxis in a medical ward: room for improvement. $J$ Gen Intern Med. 2002;17:788-791.

8. Elis A, Ellis MH. Preventing venous thromboembolism in acute medical patients. QJM. 2004;97:797-801.

9. Stark JE, Kilzer WJ. Venous thromboembolic prophylaxis in hospitalized medical patients. Ann Pharmacother. 2004;38:36-40.

10. Abba AA, Al Ghonaim MA, Rufai AM. Physicians' practice for prevention of venous thromboembolism in medical patients. J Coll Physicians Surg. 2004;14:211.

11. Chopard P, Dorffler-Melly J, Hess U, et al. Venous thromboembolism prophylaxis in acutely ill medical patients: definite need for improvement. J Intern Med. 2005;257:352-357.

12. Ageno W, Squizzato A, Ambrosini F, et al. Thrombosis prophylaxis in medical patients: a retrospective review of clinical practice patterns. Haematologica. 2002;87:746- 750.

13. Cohen AT, Tapson VF, Bergmann F, et al., for the ENDORSE Investigators. Venous thromboembolism risk and prophylaxis in the acute hospital care setting (ENDORSE Study): a multinational cross-sectional study. Lancet. 2008;371:387- 394.

14. Tapson VF, Decousus H, Pini M, et al., for the IMPROVE Investigators. Venous thromboembolism prophylaxis in acutely ill hospitalized medical patients: findings from the International Medical Prevention Registry on Venous Thromboembolism. Chest. 2007;132:936-945.

15. Giacomini M, Cook DJ, for the Evidence-Based Medicine Working Group. Users' guides to the medical literature. XXIII. Qualitative research in health care. A. Are the results of the study valid? JAMA. 2000;284:357-362. 
16. Strauss A, Corbin J. Basics of Qualitative Research: Techniques and Procedures for Developing Grounded Theory. 2nd ed. London: Sage Publications; 1998.

17. Canadian Institutes of Health Research, Natural Sciences and Engineering Research Council of Canada, Social Sciences and Humanities Research Council of Canada. Tri-Council Policy Statement: Ethical Conduct for Research Involving Humans. Ottawa, Ontario: Public Works and Government Services Canada; 1998 [with 2000, 2002, and 2005 amendments].

18. Cabana MD, Rand CS, Powe NR, et al. Why don't physicians follow clinical practice guidelines? JAMA. 1999;282:1458-1465.

19. Bradley EH, Holmboe ES, Mattera JA, Roumanis SA, Radford MJ, Krumholz HM. A qualitative study of increasing $\beta$-blocker use after myocardial infarction: why do some hospitals succeed? JAMA. 2001;285:2604-2611.

20. Sinuff T, Kahnamoui K, Cook DJ, Giacomini M. Practice guidelines as multipurpose tools: a qualitative study of noninvasive ventilation. Crit Care Med. 2007;35:776-782.
21. Tooher R, Middleton P, Pham C, et al. A systemic review of strategies to improve prophylaxis for venous thromboembolism in hospitals. Ann Surg. 2005;241:397-415.

22. Ellrodt G, Glasener R, Cadorette B, et al. Multidisciplinary rounds: an implementation system for sustained improvement in the American Heart Association's "Get With the Guidelines" Program. Crit Pathw Cardiol. 2007;6:1016-1116.

23. Cook DJ, Montori VM, McMullin JP, Finfer SR, Rocker GM. Changing clinician behaviour to improve patient safety. Lancet. 2004;363: $1224-1230$

24. Greenhalgh T, Taylor R. Papers that go beyond numbers: qualitative research. BMJ. 1997;315:740-748.

25. Kucher N, Koo S, Quiroz R, Cooper JM, Paterno MD, Soukonnikov B, Goldhaber SZ. Electronic alerts to prevent venous thromboembolism among hospitalized patients. N Engl J Med. 2005;352(10): 969-977. 